\title{
Symmetry superposition studied by surface second-harmonic generation
}

\author{
G. Lüpke, G. Marowsky, and R. Steinhoff \\ Max-Planck-Institut für Biophysikalische Chemie, Abteilung Laserphysik, Am Fassberg, \\ D-3400 Göttingen, Federal Republic of Germany \\ A. Friedrich, B. Pettinger, and D. M. Kolb \\ Fritz-Haber-Institut der Max-Planck-Gesellschaft, Faradayweg 4-6, D-1000 Berlin 33, Federal Republic of Germany \\ (Received 5 September 1989)
}

\begin{abstract}
The components of a third-rank $\chi^{(2)}$ tensor have been split into contributions due to 1-fold, 2fold, 3-fold, and $\infty$-fold or isotropic rotation axes for a surface of $C_{s}$ symmetry. Theoretical analysis of the rotation patterns obtained by the surface second-harmonic ( $\mathrm{SH}$ ) generation indicates that a complete symmetry analysis cannot be performed without knowledge of the relevant distribution functions. Rotation axes of lower symmetry create via "overtones" or "harmonics" contributions apparent in the analysis of the rotation axes of higher symmetry. An experimental example is the observation of structural changes of $\mathrm{Au}(111)$ surfaces in an aqueous electrolytic environment. Potential-dependent buildup and removal of a $\mathrm{Au}(111)-(1 \times 23)$ surface could be monitored in situ and in real time. Symmetry analysis of the $\mathrm{SH}$ rotation patterns reveals both contributions due to a 3 -fold axis due to the regular $(1 \times 1)$ structure and simultaneously a 1 -fold and a 2 -fold axis due to the $(1 \times 23)$ reconstruction.
\end{abstract}

\section{INTRODUCTION}

The various techniques provided by nonlinear optics are attractive methods for symmetry analysis of crystalline bulk and surface structures. In particular, the second-harmonic generation (SHG), with its high intrinsic surface specifity, has found numerous applications in interface analyses, such as detection of adsorbates, anisotropy analysis, reconstruction processes under UHV conditions, or time-resolved observation of structural changes. ${ }^{1-4}$ Because of its unique in situ applicability, optical second-harmonic generation is particularly attractive for studies of electrode surface modifications within an electrochemical cycle. ${ }^{5}$ Nonlinear-optical experiments involving electrochemical processes were mostly restricted in the past to the documentation of the changes in rotational anisotropy via SHG of the crystalline electrode itself upon variation of the experimental conditions, such as bias potential, ${ }^{6,7}$ or to the determination of the structure of an electrodeposited overlayer. ${ }^{8}$ In the latter case a monolayer of $\mathrm{Cd}$ on a $\mathrm{Ag}(111)$ surface generated in an underpotential deposition process served as a source of the observed anisotropic SH signal. Modifications of the strength and the symmetry of the SH response of crystalline samples exposed to overlayer adsorption under UHV conditions were the main result of various experimental approaches with surfaces of $\langle 111\rangle$ direction: Tom et al. ${ }^{9}$ demonstrated adsorption of $\mathrm{O}_{2}, \mathrm{CO}$, and $\mathrm{Na}$ on $\mathrm{Rh}(111)$, and Anderson and Hamilton studied the effect of $\mathrm{CO}$ and $\mathrm{H}_{2}$ adsorption on $\mathrm{Ni}(111)$ surfaces. ${ }^{10,11}$ It is the purpose of this contribution to unravel, both in theory and experiment, the complications that arise if $\mathrm{SH}$ signals, well defined in terms of their symmetry character, superimpose. An experimental example is a potential-induced reconstruction of a $(1 \times 23)$ overlayer on a $\mathrm{Au}(111)$ surface within a certain potential range established previously in Ref. 12. In the reconstruction regime we expect simultaneously $\mathrm{SH}$ contributions from the top layer of $\mathrm{AU}(111)$ of $C_{3 v}$ symmetry $^{13}$ and from the superstructure of $C_{s}$ symmetry. ${ }^{14}$

\section{THEORY}

Our approach to surface-symmetry analysis is an extension of the usual procedure in nonlinear optics, polarization-dependent excitation with a fundamental of frequency $\omega$, and polarization-dependent registration of the resultant SH signal of frequency $2 \omega$ upon rotation of the sample about the surface normal. According to Fig. 1 , we denote this direction with the $z$ axis. The $x-y$ coordinates define the surface plane with the $y$ direction coinciding with the direction for $s$ polarization and $p$ polarization coinciding with the plane of incidence, the $x-z$ plane. In order to include rotation-angle independent (isotropic) contributions, we consider irradiation of the samples under an oblique angle of incidence, typically $45^{\circ}$. The basic idea of the mathematical procedure is to consider the symmetry of the surface arrangement and to split the respective tensor into contributions representing the various symmetry axes. As will be discussed in detail in the experimental section, analysis of these symmetryresolved tensor components provides information as to lateral and horizontal configurational changes induced by electrode potential variation. The SH response of a surface layer can be written in dipole approximation as ${ }^{15}$

$$
P_{i}(2 \omega)=\chi_{i j k}: E_{j}(\omega) E_{k}(\omega),
$$

where $i, j, k$ denote vector components with respect to the 
$x-y-z$ coordinate system and the sum convention rule applies. The fundamental field is $E(\omega)$ and $\chi_{s}^{(2)}=\left\{\chi_{i j k}\right\}$ denotes the third-rank susceptibility tensor, which reflects the symmetry of the surface. Rather than in the most general case of triclinic $\left(C_{1}\right)$ symmetry and 18 nonvanishing components, ${ }^{16}$ the superposition of $C_{s}$ and $C_{3 v}$ symmetry will exhibit monoclinic $C_{s}$ symmetry and therefore includes one mirror plane. According to Ref. 16 we expect for $C_{s}$ symmetry in standard orientation
( $m \perp y$ axis) in contracted notation ${ }^{17}$ the following tensor with 10 independent elements:

$$
\chi^{(2)}=\left(\begin{array}{llllll}
\chi_{x x x} & \chi_{x y y} & \chi_{x z z} & & \chi_{x z x} & \\
& & & \chi_{y z y} & & \chi_{y x y} \\
\chi_{z x x} & \chi_{z y y} & \chi_{z z z} & & \chi_{z z x} &
\end{array}\right) .
$$

The quantities $\left\{d_{11}^{(1)}, \ldots, d_{33}^{(\infty)}\right\}$ represent rotation amplitudes of the respective 1 -fold, 2 -fold, 3 -fold, and $\infty$-fold symmetry axes. In total, the different rotation axes are represented by the following set of amplitudes occurring in tensor (3):

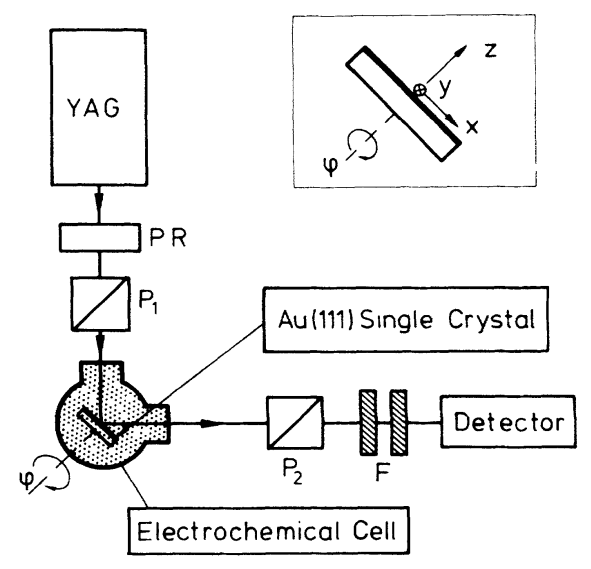

FIG. 1. Experimental setup for SHG observation from $\mathrm{Au}$ samples within electrochemical cell. PR: Polarization rotator; $P_{1}, P_{2}$ : polarizers; $F$ : filter for rejection of the fundamental wavelength at $1064 \mathrm{~nm}$.

$$
\left.\begin{array}{l}
\left.\begin{array}{l}
d_{11}^{(1)} \\
d_{12}^{(1)} \\
d_{13}^{(1)} \\
d_{16}^{(1)} \\
d_{34}^{(1)}
\end{array}\right\} 1 \text {-fold axis with } d_{11}^{(1)}=d_{12}^{(1)}+2 d_{16}^{(1)}, \\
\left.\begin{array}{l}
d_{14}^{(2)} \\
d_{31}^{(2)}
\end{array}\right\} \text { 2-fold axis }, \\
\left.d_{11}^{(3)}\right\} 3 \text { - fold axis }, \\
d_{15}^{(\infty)} \\
d_{31}^{(\infty)} \\
d_{33}^{(\infty)}
\end{array}\right\} \infty \text {-fold axis . }
$$

The reason for the selection of $C_{s}$ symmetry was the experimental observation that the rotation amplitudes $d_{11}^{(1)}$ and $d_{11}^{(3)}$ have identical phases. It is obvious from tensor (3) that at a given position always amplitudes of odd or even rotational symmetry group together. In particular, projections in the direction of the surface normal contain necessarily the amplitudes of the 2-fold and $\infty$-fold axes and the amplitudes $d_{13}^{(1)}$ and $d_{34}^{(1)}$ of the 1 -fold axis. Experimentally, we have determined by Fourier analysis of the $\mathrm{SH}$ rotation patterns with respect to azimuthal angle $\phi$ all relevant amplitudes introduced in tensor (3). Comparison of rotation amplitudes related to different polarization conditions required consideration of all linear and nonlinear Fresnel factors of the gold surface. ${ }^{18,19}$ 
Next, we would like to show that analysis of a surface structure always leads to apparent contributions at higher symmetry due to amplitudes of lower symmetry. As an example, we present the effect of the 1-fold axis, characterized by its amplitude $d_{12}^{(1)}$, onto the evaluation of a 3fold degree of symmetry described by the amplitude $d_{11}^{(3)}$. This "overtone" contribution strongly depends on the distribution function responsible for the lower symmetry. It may happen that a SHG experiment leads to the determination of a 3-fold symmetry contribution in total absence of any 3-field rotation axis of the real sample. To facilitate the discussion, we would like to symbolize the nonlinearity expressed by the $\chi^{(2)}(\phi)$ tensor by a single, dominant component located for the sake of simplicity in the $x-y$ surface plane (cf. Fig. 2). For a general, threedimensional distribution function $F(\phi, \theta)$, with $\phi$ the already defined azimuthal angle and $\theta$ the tilt angle with respect to the surface normal, this means that $F(\phi, \theta)$ reduces to $F(\phi)$ and tensor (3) will maintain only rotation axes of an odd degree of symmetry. These rotation amplitudes can be derived from the distribution function as follows:

$$
\begin{aligned}
& d_{11}^{(3)}=\frac{1}{16} \int_{0}^{2 \pi} F(\phi) \cos 3 \phi d \phi, \\
& d_{12}^{(1)}=\frac{1}{16} \int_{0}^{2 \pi} F(\phi) \cos \phi d \phi .
\end{aligned}
$$

Next, let us define the autocorrelation function ${ }^{20}$

$$
R\left[F, F\left(\phi_{0}\right)\right]=\frac{1}{2 \pi} \int_{0}^{2 \pi} F(\phi) F\left(\phi+\phi_{0}\right) d \phi,
$$

which leads to the degree of symmetry of the distribution, characterized by $F(\phi)$ :

$$
\begin{aligned}
& d^{(1)}=R\left[F, F\left(0^{\circ}\right)\right]-R\left[F, F\left(120^{\circ}\right)\right], \\
& d^{(3)}=R\left[F, F\left(120^{\circ}\right)\right] .
\end{aligned}
$$

With reference to Fig. 2 and Table I, we would like to discuss the implications of Eqs (5)-(8) for two selected distribution functions: $F^{(1)}(\phi)=a \cos \phi$ (upper panel of Fig. 2), and $F^{(1)}(\phi)=a \delta(\phi)$ as shown in the lower part of this figure. The right-hand part of Fig. 2 shows the changes in the respective distribution function $F^{(1),(3)}(\phi)$ upon addition of $F^{(1)}(\phi)$ onto the corresponding distribution function $F^{(3)}(\phi)=b \cos (3 \phi)$ and

$$
F^{(3)}(\phi)=b\left[\delta(\phi)+\delta\left(\phi+120^{\circ}\right)+\delta\left(\phi-120^{\circ}\right)\right],
$$

characterizing a 3-fold rotation axis of constant amplitude $b$. A comparison of the upper and lower panel of
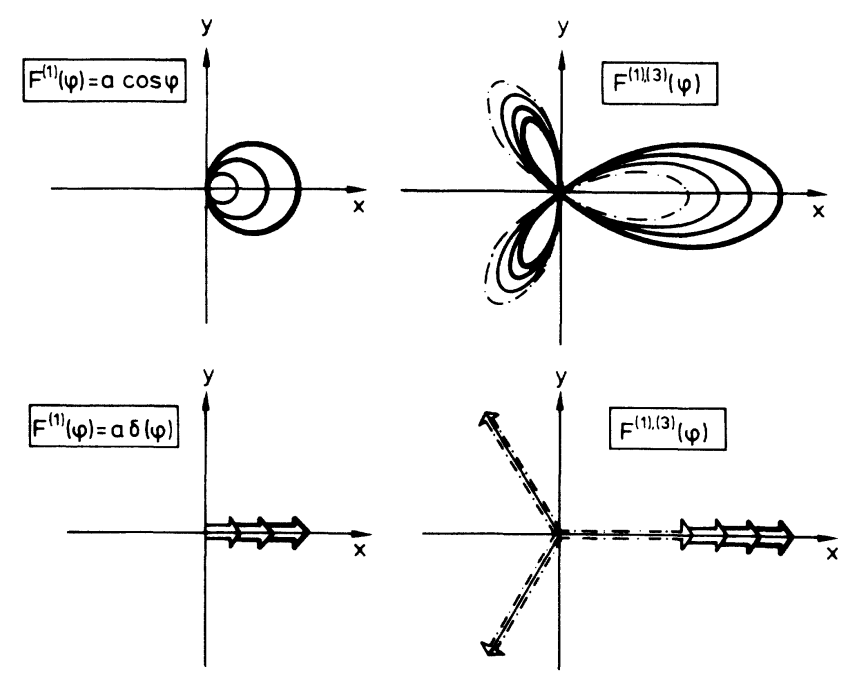

FIG. 2. Superposition of 1 -fold and 3-fold symmetries for two selected distribution functions (for details see the text).

Fig. 2 and reference to Table I shows that an increasing contribution of a 1 -fold symmetry axis leads for the two selected distribution functions to quite different results.

In the case of the cos distribution, the nonlinear SHG technique and the autocorrelation function monitor, in both cases, the amplitudes $a$ and $b$ from the 1-fold and 3fold contribution. The autocorrelation function is a power spectrum, so that the amplitudes $a$ and $b$ appear squared. The rotation amplitudes of tensor (3) can be expressed as the values of the cross-correlation functions of the distribution functions $F^{(1),(3)}(\phi)$ and the cos functions at zero-angle displacement:

$$
\begin{aligned}
d_{12}^{(1)} & =\frac{\pi}{8} R\left[\cos \phi, F^{(1),(3)}\left(\phi_{0}=0^{\circ}\right)\right] \\
& =\frac{\pi}{8} R\left[\cos \phi, F^{(1)}\left(\phi_{0}=0^{\circ}\right)\right], \\
d_{11}^{(3)} & =\frac{\pi}{8}\left[\cos 3 \phi, F^{(1),(3)}\left(\phi_{0}=0^{\circ}\right)\right] \\
& =\frac{\pi}{8} R\left[\cos 3 \phi, F^{(3)}\left(\phi_{0}=0^{\circ}\right)\right] .
\end{aligned}
$$

In this special case

$$
\begin{aligned}
& R\left[\cos \phi, F^{(3)}\left(\phi_{0}=0^{\circ}\right)\right]=0, \\
& R\left[\cos 3 \phi, F^{(1)}\left(\phi_{0}=0^{\circ}\right)\right]=0,
\end{aligned}
$$

TABLE I. Results for $R$ and SHG for two types of distribution functions.

\begin{tabular}{ccc}
\hline \hline $\begin{array}{c}\text { Type of distribution } \\
\text { function }\end{array}$ & $R\left[F^{(1)}, F^{(1)}\right]+R\left[F^{(3)}, F^{(3)}\right]$ & $\begin{array}{c}\text { SHG } \\
d^{(1)}: d^{(3)}\end{array}$ \\
\hline$F^{(1),(3)}(\phi)=a \cos \phi+b \cos 3 \phi$ & $a^{2} / b^{2}$ & $a / b$ \\
$F^{(1),(3)}(\phi)=a \delta(\phi)+b \delta(\phi)$ & & \\
$+b\left[\delta\left(\phi+120^{\circ}\right)+\delta\left(\phi-120^{\circ}\right)\right]$ & $a^{2} / 3 b^{2}$ & $a /(a+3 b)$ \\
\hline \hline
\end{tabular}


and hence the rotation amplitude of tensor (3) are completely decoupled.

The situation is different for the $\delta$ distribution (lower panel of Table I). The contribution of the cross correlation $R\left[\cos \phi, F^{(3)}\left(\phi_{0}=0^{\circ}\right)\right]$ vanishes for the 1 -fold rotation amplitude $d_{12}^{(1)}$, but the 3-fold rotation amplitude $d_{11}^{(3)}$ contains a nonvanishing contribution of the cross correlation $R\left[\cos 3 \phi, F^{(1)}\left(\phi_{0}=0^{\circ}\right)\right]$ and thus an additional term due to the 1-fold axis ("overtone" or "harmonic"). Even for $b=0$ one might be tempted to interpret a rotation amplitude $d_{11}^{(3)}=d_{12}^{(1)}$ as 3-fold symmetry existing in reality. So far we have restricted the discussion to distributions within the $x-y$ surface plane. If the nonlinear dipole is tilted by an angle $\theta$ with respect to the $z$ axis, additional amplitudes representing even degrees of symmetry occur. We expect a 2 -fold axis and due to the limited symmetry-resolving power of a third-rank tensor the 4fold axis cannot be differentiated from an $\infty$-fold or isotropic axis. The amplitudes $d_{14}^{(2)}$ and $d_{13}^{(1)}$ can be derived in a similar way from $F(\phi, \theta)$ :

$$
\begin{aligned}
& d_{14}^{(2)}=\frac{1}{4} \int_{0}^{\pi} \int_{0}^{2 \pi} F(\phi, \theta) \sin ^{2} \theta \cos \theta \cos 2 \phi d \phi d \phi, \\
& d_{13}^{(1)}=\frac{1}{4} \int_{0}^{\pi} \int_{0}^{2 \pi} F(\phi, \theta) \sin \theta \cos ^{2} \theta \cos \phi d \phi d \theta .
\end{aligned}
$$

For a $\delta$-type distribution with respect to $\phi$ with

$$
F(\phi, \theta)=F^{(1)}(\phi) F^{(1)}(\theta)+F^{(3)}(\phi) F^{(3)}(\theta),
$$

Eqs. (11) and (12) yield the following expressions:

$$
\begin{aligned}
& d_{14}^{(2)}=\frac{1}{4} a_{\phi} \int_{0}^{\pi} F^{(1)}(\theta) L_{1}(\cos \theta) d \theta \\
& d_{13}^{(1)}=\frac{1}{4} a_{\phi} \int_{0}^{\pi} F^{(1)}(\theta) L_{1}(\sin \theta) d \theta
\end{aligned}
$$

The quantity $L_{1}(u)$ denotes the difference between the first and third Legendre polynomial: ${ }^{21}$

$$
L_{1}(u)=\frac{2}{5}\left[P_{1}(u)-P_{3}(u)\right] .
$$

Figure 3 shows examples of the influence of the weighting factors derived from Eqs. (14)-(16) for the various rotation amplitudes.

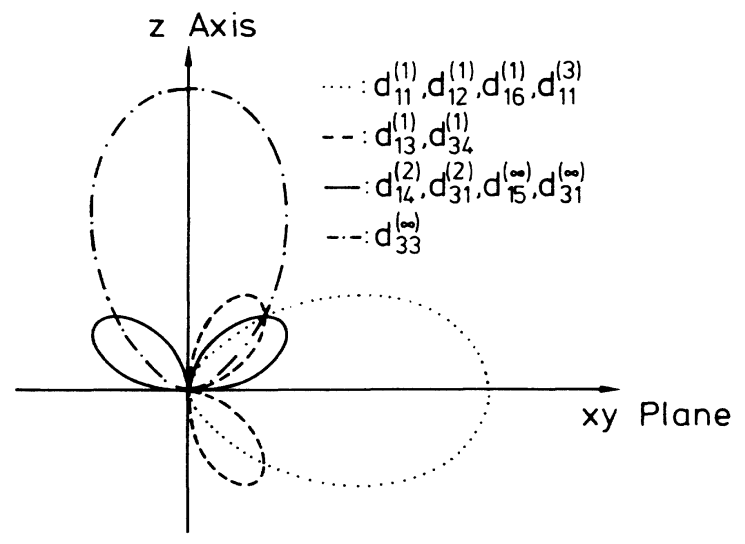

FIG. 3. Spatial weighting factors for the various rotation amplitudes of tensor (3) according to Eqs. (14) - (16).
For a $\delta$-type distribution

$$
F^{(1)}(\theta)=a_{\theta} \delta\left(\theta-\theta_{0}\right)
$$

the angle $\theta_{0}$ can be obtained from the ratio $d_{14}^{(2)} / d_{13}^{(1)}$.

$$
\frac{d_{14}^{(2)}}{d_{13}^{(1)}}=\frac{a_{\phi} a_{\theta} \sin ^{2} \theta_{0} \cos \theta_{0}}{a_{\phi} a_{\theta} \sin \theta_{0} \cos ^{2} \theta_{0}}=\tan \theta_{0} .
$$

The latter result is similar to the procedure applied for isotropic surface-layer distributions, where an average tilt angle is derived by the relation ${ }^{22}$

$$
\frac{d_{15}^{(\infty)}}{d_{33}^{(\infty)}}=\frac{1}{2} \tan ^{2} \theta_{0}
$$

\section{EXPERIMENTAL}

As shown in Fig. 1, all SHG measurements were made in reflection under an angle of incidence of $45^{\circ}$. Laser lightsource is the fundamental output of a $10-\mathrm{Hz} \mathrm{Nd}$ YAG laser, delivering $10-\mathrm{mJ}$ pulses of $10-\mathrm{ns}$ duration, which were slightly focused to $1 \mathrm{~mm}^{2}$ onto the singlecrystal Au electrode within an electrochemical cell. Appropriate polarizers and polarization rotators for the incident fundamental and the resultant $\mathrm{SH}$ radiation were applied in order to single out well-defined combinations of tensor components of the susceptibility tensor (3). Further details of the optical arrangement are published elsewhere. $^{23}$ The gold electrode was a disc of about 4 $\mathrm{mm}$ in diameter and 2-3 $\mathrm{mm}$ thick. After mechanical and electrochemical polishing, the crystal was subjected to a flame treatment. An electrolyte served as an aqueous solution of $0.01-\mathrm{M} \mathrm{H}_{2} \mathrm{SO}_{4}$. All potentials are quoted with respect to the saturated calomel electrode $\left(V_{\mathrm{SCE}}\right)$.

\section{RESULTS}

In Ref. 12 it has been demonstrated that the $\mathrm{Au}(111)$ $(1 \times 23)$ reconstruction is stable within a certain potential range of the double-layer charging region. The ex situ identification of the surface morphology, requiring immersion of a flame-treated and reconstructed surface into the electrolyte and emersion after a potential excursion into the oxide formation region with removal of the reconstruction, however, did not permit to establish well-defined stability limits due to the inherent difficulties of an analysis outside the electrochemical cell. Application of in situ diagnostics via SHG clearly revealed that the $(1 \times 23)$ reconstruction starts to be discernible at 1.0 $V_{\mathrm{SCE}}$ and grows to the cathodic limit at $-0.5 V_{\mathrm{SCE}} \cdot{ }^{23}$ Our subsequent symmetry analysis addresses to this potential range.

Figure 4(a), 4(b), and 4(c) show the s-polarized $\mathrm{SH}$ intensities for $p$ - and $s$-polarized illumination, as the $\mathrm{Au}(111)$ crystal was rotated $360^{\circ}$ about the surface normal. The origin of the angular scale was in $\langle 100\rangle$ direction and is the same for all the figures here. In Fig. 5(a) the $\mathrm{Au}$ electrode was potentiostated at $+0.7 V_{\text {SCE }}$ and the 3-fold symmetry of the unreconstructed $\mathrm{Au}(111)$ $(1 \times 1)$ surface is clearly reflected in the rotational anisotropy of the SH intensity, given by 


$$
I_{p s}(2 \omega) \propto\left|d_{11}^{(3)} \sin 3 \phi\right|^{2} .
$$

In this case, the pattern reveals a 6-fold symmetry with evenly spaced maxima separated by $60^{\circ}$. For potentials anodic by $+0.2 V_{\mathrm{SCE}}$ the angular rotation pattern of the SH signals, shown in Fig. 4(b), is distinctly different. A 1 -fold symmetry pattern is superimposed onto a 3-fold one, indicating the $\mathrm{Au}(1 \times 23)$ superstructure. For $s$ polarized excitation and s-polarized detection two dominant peaks at $90^{\circ}$ and $270^{\circ}$ appear and the other four peaks are markedly reduced in their intensity, corresponding to a superposition of 1 -fold and 3-fold symmetry elements:

$$
I_{s s}(2 \omega) \propto\left|d_{11}^{(1)} \sin \phi-d_{11}^{(3)} \sin 3 \phi\right|^{2} .
$$

Comparison of Fig. 4(a) and 4(b) demonstrates that the surface structural transition between $(1 \times 1)$ and $(1 \times 23)$ is exceptionally clearly seen in the rotational anisotropy of the SH signal, although there is only a $4 \%$ compression of the top layer in the latter case. Figure 4(b) demonstrates the symmetry change of the Au(111) surface in the $x y$ plane. A symmetry analysis respective to the surface normal requires consideration of the 2 -fold and $\infty$-fold rotation amplitudes, because these components appear only if at least one component of $E(\omega)$ [cf. Eq. (1)] is po-
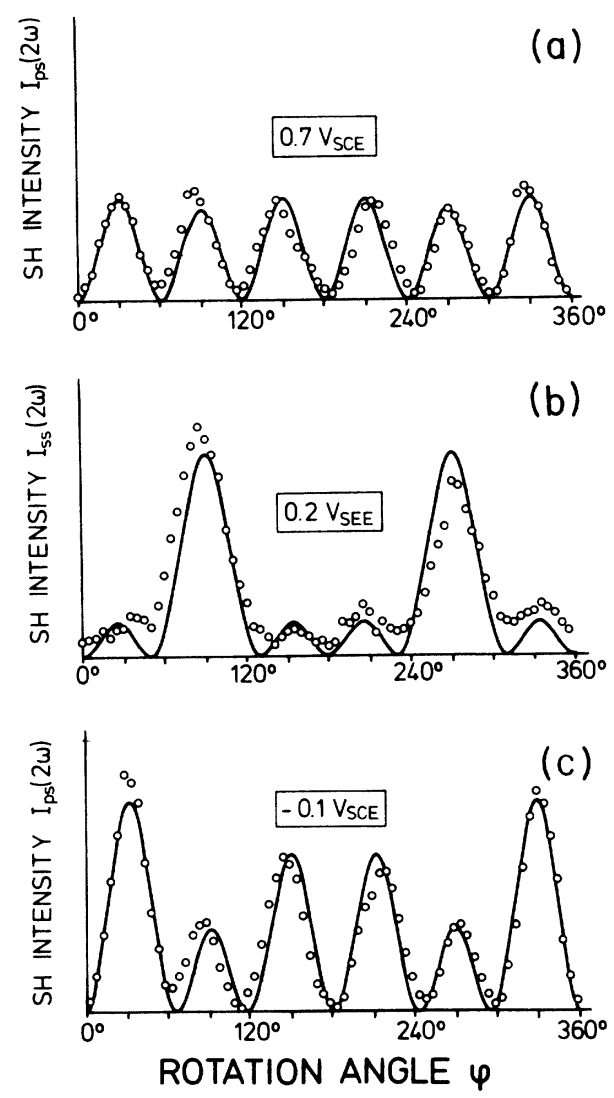

FIG. 4. SH intensity vs rotation angle for three values of bias potential. Compilation shows experimental data points and least-square fits according to Eqs. (19) -(21).

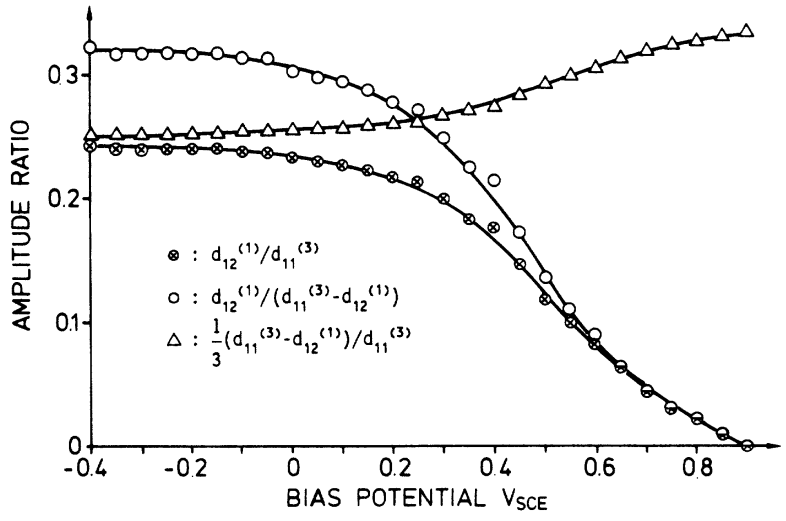

FIG. 5. Amplitude ratios as defined by symbols shown in inset vs bias potential evaluated under the two assumptions of a $\delta$ type of cos-type distribution function (for details see the text).

larized in the $z$ direction. This condition is fulfilled for p-polarized incident radiation. Figure 4(c) shows the rotation dependent $s$-polarized $\mathrm{SH}$ intensity at $-0.1 \mathrm{~V}$, given by

$$
\begin{aligned}
I_{p s}(2 \omega) \propto \mid & f_{x}^{2}\left(d_{12}^{(1)} \sin \phi+d_{11}^{(3)} \sin 3 \phi\right) \\
& +f_{z}^{2} d_{13}^{(1)} \sin \phi+\left.f_{x} f_{z} d_{14}^{(2)} \sin 2 \phi\right|^{2}
\end{aligned}
$$

with $f_{x}, f_{z}$ the linear Fresnel factors. ${ }^{19}$ From Fig. 4(c) an additional 2-fold rotation amplitude $d_{14}^{(2)}$ can be derived and a ratio of $d_{14}^{(2)} / d_{11}^{(3)}=0.05$ is determined by Fourier analysis according to Eq. (20). The 3-fold rotation amplitude $d_{11}^{(3)}$ is found to be constant when the $\mathrm{Au}(111)$ electrode is polarized between the limits of the double-layer charging region. This allows to monitor in situ the structural transition by recording the $\mathrm{SH}$ intensity at either $\phi=90^{\circ}$ or $\phi=270^{\circ}$ as a function of electrode potential, derived from Eq. (20):

$$
I_{s s}(2 \omega) \propto\left|1+d_{12}^{(1)} / d_{11}^{(3)}\right|^{2} .
$$

Figure 5 shows the potential dependence of the following amplitude ratios evaluated for the two types of distribution functions listed in Table I:

$$
\begin{aligned}
& d_{12}^{(1)} / d_{11}^{(3)}, \\
& d_{12}^{(1)} /\left(d_{11}^{(3)}-d_{12}^{(1)}\right), \\
& \frac{1}{3}\left(d_{11}^{(3)}-d_{12}^{(1)}\right) / d_{11}^{(3)} .
\end{aligned}
$$

The increase of $d_{12}^{(1)}$ with (negative) potential directly reflects the raise of the $(1 \times 23)$ reconstruction $(\otimes$ symbols) for the example of the cos distribution with no overtone contribution to the 3-fold symmetry, the experimentally observed constancy of the 3 -fold rotation amplitude on applied bias potential. If an overtone contribution has to be taken into account for a $\delta$-type distribution function, the rise of the reconstruction with potential is even stronger ( $\bigcirc$ symbols), and the "true" amplitude of the 3fold rotation axis is no longer constant ( $\triangle$ symbols). The potential dependence of the SH signal also reveals that the 1 -fold symmetry pattern rapidly decreases for poten- 
tials exceeding $+0.4 V_{\mathrm{SCE}}$, indicating that the $(1 \times 23)$ reconstruction is stable only up to that value, whereas no cathodic limit for the stability range of the $(1 \times 23)$ structure was found. For $U>0.7 V_{\mathrm{SCE}}$, the $(1 \times 23) \rightarrow(1 \times 1)$ transition is completed and the $\mathrm{Au}(111)$ surface is now unreconstructed.

\section{DISCUSSION}

Before discussing our results in detail, we note that the observed SH signals do in fact arise from the surface layer of the sample. Attempted measurements of the bulk anisotropic contributions by $s$-polarized signals from an unreconstructed $\mathrm{Au}(100)$ face failed, indicating that the higher-order magnetic dipole and electric quadrupole terms in the bulk are not significant at the pump wavelength (1064 $\mathrm{nm})$.

The surface layer of a (111) face of a cubic crystal such as gold has $C_{3 v}$ symmetry. ${ }^{13}$ The top atomic layer of the perfectly terminated $(1 \times 1)$ surface is hexagonally close packed and therefore $C_{6 v}$ symmetric, but if one includes the second or more atomic layers it is clear that the surface potential for the surface electrons should be $C_{3 v}$ symmetric. Reconstruction of the $\mathrm{Au}(111)$ face into $(1 \times 23)$, which represents a hexagonal close-packed structure with an uniaxial compression of about $4 \%$ in $\langle 110\rangle$ direction (Fig. 6), leads to a $C_{s}$ symmetry superimposed onto $C_{3 v}$ symmetry. It should be noted that the measured rotation pattern from a macroscopic surface is a superposition of signals from the three domains of the unreconstructed $\mathrm{Au}(111)$ surface, since there are three alternatives in which the uniaxially reconstructed region can align with the second layer. From our experimental results we are led to the conclusion that various $(1 \times 23)$

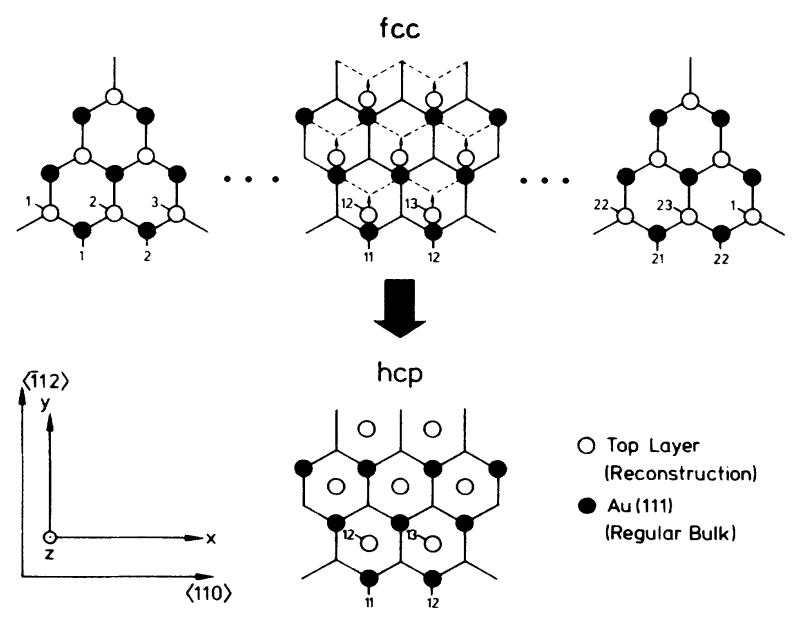

FIG. 6. Schematic of $(1 \times 23)$ reconstruction shown by representation of three selected surface regimes of fcc structure (upper panel) and "relaxation" into hcp structure (center of lower panel). Position of crystallographic coordinate system with respect to $x-y-z$ system is also shown.

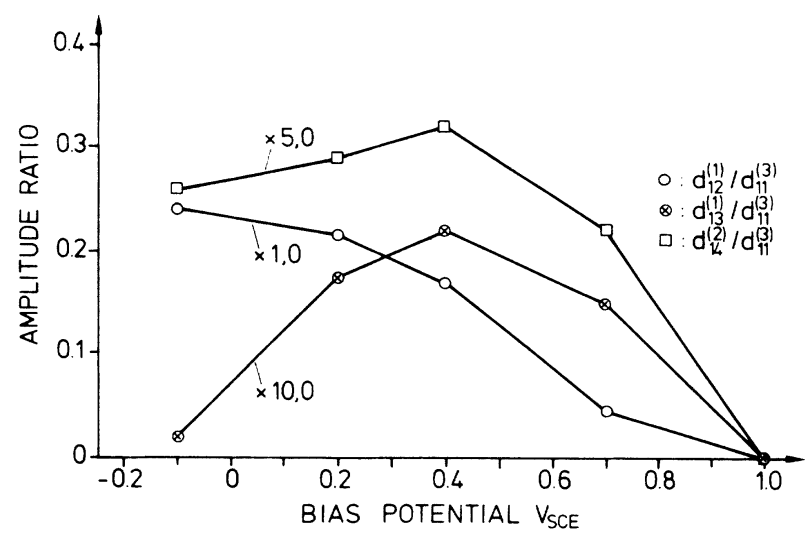

FIG. 7. Amplitude ratios as defined by symbols shown in inset vs bias potential. The $d_{11}^{(3)}$ dependence was taken from Fig. 5 ( $\Delta$ symbols under consideration of a $\delta$-type distribution). The $y$ axis shows proper scale for $d_{12}^{(1)} / d_{11}^{(3)}$ ratio. Multiplication factors for other ratios are indicated.

domains are not equally distributed, but have a preferential orientation that could be introduced by, e.g., atomic steps. Experimental results by Takayanagi and Yagi suggest that the compression in $\langle 110\rangle$ direction is not uniform, but localized in two transition regions where the stacking changes abruptly from the normal fcc termination to an hcp termination. ${ }^{24}$ But this fails to explain the high 1 -fold rotation amplitude $d_{11}^{(1)}$, because the hcp termination reduces only the 3 -fold rotation amplitude $d_{11}^{(3)}$. Harten et al. present a refined model, where the transition between fcc and hcp regions is described by a gradual $\langle 110\rangle$-dependent shift given by a soliton. ${ }^{25}$ To account for the 1 -fold symmetry, it is necessary that the fcc and hcp regions have different size which removes the otherwise 2-fold rotation symmetry of the surface layer.

The potential dependence of the odd rotation amplitudes $d_{11}^{(3)}$ and $d_{12}^{(1)}$, shown in Fig. 5, yields only information about the dynamic of the transformation in the surface plane. Figure 6 indicates that as a result of the termination of the reconstruction the overlayer structure "relaxes" from the fcc structure into the hcp structure. This behavior can be substantiated by a detailed study of the potential dependence of the relevant rotation amplitudes. Figure 7 summarizes the experimental findings in a plot of $d_{12}^{(1)}$ (○ symbols), $d_{13}^{(1)}\left(\otimes\right.$ symbols), and $d_{14}^{(2)}(\square$ symbols) versus bias potential with all rotation amplitudes normalized to $d_{11}^{(3)}$. For its potential dependence, the behavior for a $\delta$-type distribution was considered. The amplitudes $d_{14}^{(2)}$ and $d_{13}^{(1)}$ reflect structural changes with respect to the direction of the surface normal. These amplitudes possess relatively large values in the anodic region at $+0.7 V_{\mathrm{SCE}}$, where the conversion from the metastable $(1 \times 23)$ reconstruction to the equilibrium $(1 \times 1)$ reconstruction occurs. This reflects the fact that $\mathrm{Au}$ atoms need some degree of freedom upon motion in the $z$ direction before a compression in the $\langle 110\rangle$ direction takes place. The similar behavior of the two components $d_{13}^{(1)}$ and $d_{14}^{(2)}$ indicates that the 2 -fold rotation amplitude $d_{14}^{(2)}$ is primarily a harmonic contribution of the 1-fold symmetry, in good agreement with the assump- 
tion of a $\delta$ distribution. The strong decrease of the $d_{13}^{(1)}$ component in the cathodic region from $+0.2 V_{\mathrm{SCE}}$ to $-0.1 V_{\mathrm{SCE}}$ can be explained, as the previously mentioned relaxation of the gold atoms in the unoccupied hcp sites, so that the nonlinear moment finally occupies the surface plane in agreement with a maximum of the $d_{12}^{(1)}$ component.

We have utilized the sensitivity of the SHG process with respect to the degree of symmetry of a rotation axis to monitor in situ the surface transformation from the $(1 \times 1)$ to the $(1 \times 23)$ reconstruction. It is also possible to monitor the appearance or the disappearance of a mirror plane. In that way Heinz et al. demonstrated the rise of an additional mirror plane during a heating circle, indicating the transformation from the $(2 \times 1)$ reconstruction to the $(7 \times 7)$ reconstruction of a silicon (111) surface. ${ }^{3} \mathrm{~A}$ main disadvantage of this method is, that the surface normal lies in the mirror plane, so that no information can be obtained about the dynamic of the transformation respective to the surface normal. This information is given by a separation of the tensor elements in their rotation amplitudes.

\section{CONCLUSIONS}

We have shown, both in theory and experiment, the complication that arise if SH signals, well defined in terms of their symmetry character, superimpose. A complete symmetry analysis cannot be performed without knowledge of the relevant distribution functions of the nonlinear dipole momentum. The dynamic behavior of the 1-fold, 2-fold, and 3-fold rotation amplitudes was used to demonstrate the power of SHG for monitoring in situ changes in the surface structure. For the reconstructed $\mathrm{Au}(111)-(1 \times 23)$ surface, a pronounced 1-fold symmetry pattern was observed in the SHG intensity as expected for a surface of $C_{s}$ symmetry. The 2 -fold rotation amplitude $d_{14}^{(2)}$ is found to be mostly a "harmonic" contribution of the 1-fold symmetry. The 3 -fold rotation amplitude $d_{11}^{(3)}$ is potential independent but in the case of a $\delta$ distribution includes a contribution of the 1-fold symmetry by an "overtone" contribution. The dynamic behavior of the 1-fold $d_{13}^{(1)}$ and 2-fold $d_{14}^{(2)}$ rotation amplitudes indicate that a part of the gold atoms relax into the hcp sites and therefore reduce the nonlinear dipole momentum in the direction of the surface normal. Our symmetry and dynamic analysis of the Au reconstruction confirms the soliton model in good agreement to the high-resolution helium-atom diffraction experiments of Harten et al. ${ }^{25}$ The technique of directional analysis of a nonlinear crystalline surface layer via SHG will also be useful for studies of the atomic disorder induced by electronic excitation. ${ }^{4}$
${ }^{1}$ Y. R.Shen, J. Vac. Sci. Technol. B 3, 1464 (1985).

${ }^{2}$ H. W. K. Tom, T. F. Heinz, and Y. R. Shen, Phys. Rev. Lett. 51, 1983 (1983).

${ }^{3}$ T. F. Heinz, M. M. T. Loy, and W. A. Thompson, Phys. Rev. Lett. 54, 63 (1985).

${ }^{4}$ H. W. K. Tom, G. D. Aumiller, and C. H. Brito-Cruz, Phys. Rev. Lett. 60, 1438 (1988).

${ }^{5}$ G. L. Richmond, J. M. Robinson, and V. L. Shannon, Prog. Surf. Sci. 28, 1 (1988).

${ }^{6}$ V. L. Shannon, D. A. Koos, and G. L. Richmond, J. Phys. Chem. 91, 5548 (1987).

${ }^{7}$ H. M. Rojhantalab and G. L. Richmond, J. Phys. Chem. 93, 3269 (1989).

${ }^{8}$ J. Miragliotta and T. E. Furtak, Phys. Rev. B 37, 1028 (1988).

${ }^{9}$ H. W. K. Tom, C. M. Mate, X. D. Zhu, J. E. Crowell, T. F. Heinz, G. A. Somorjai, and Y. R. Shen, Phys. Rev. Lett. 52, 348 (1984).

${ }^{10}$ R. J. M. Anderson and J. C. Hamilton, Phys. Rev. B 38, 8451 (1988).

${ }^{11}$ J. C. Hamilton and R. J. M. Anderson, Chem. Phys. Lett. 151, 455 (1988).

${ }^{12}$ M. S. Zei, G. Lehmpfuhl, and D. M. Kolb (unpublished).

13J. E. Sipe, D. J. Moss, and H. M. van Driel, Phys. Rev. B 35, 1129 (1987).
${ }^{14}$ M. A. van Hove, R. J. Koestner, P. C. Stair, J. P. Biberian, L. L. Kesmodel, I. Bartos, and G. A. Somorjai, Surf. Sci. 103, 189 (1981); 103, 218 (1981).

${ }^{15}$ Y. R. Shen, The Principles of Nonlinear Optics (Wiley, New York, 1984).

${ }^{16}$ A. Yariv and P. Yeh, Optical Waves in Crystals (Wiley, New York, 1983).

${ }^{17}$ A. Yariv, Quantum Electronics (Wiley, New York, 1975).

${ }^{18}$ N. Bloembergen and P. S. Pershan, Phys. Rev. 128, 606 (1962).

${ }^{19}$ B. U. Felderhof and G. Marowsky, Appl. Phys. B 44, 11 (1987).

${ }^{20}$ J. S. Bendat and A. G. Piersol, Random Data: Analysis and Measurement Procedures (Wiley, New York, 1971).

${ }^{21}$ M. Abramowitz and T. A. Stegun, Handbook of Mathematical Functions (Dover, New York, 1965).

${ }^{22}$ G. Marowsky, A. Gierulski, R. Steinhoff, D. Dorsch, R. Eidenschnik, and B. Rieger, J. Opt. Soc. Am. B 4, 956 (1987).

${ }^{23}$ A. Friedrich, B. Pettinger, D. M. Kolb, G. Lüpke, R. Steinhoff, and G. Marowsky, Chem. Phys. Lett. 163, 123 (1989).

${ }^{24}$ K. Takayanagi and K. Yagi, Trans. Jpn. Inst. Met. 24, 337 (1983).

${ }^{25}$ U. Harten, A. M. Lahee, J. P. Toennies, and Ch. Wöll, Phys. Rev. Lett. 54, 2619 (1985). 


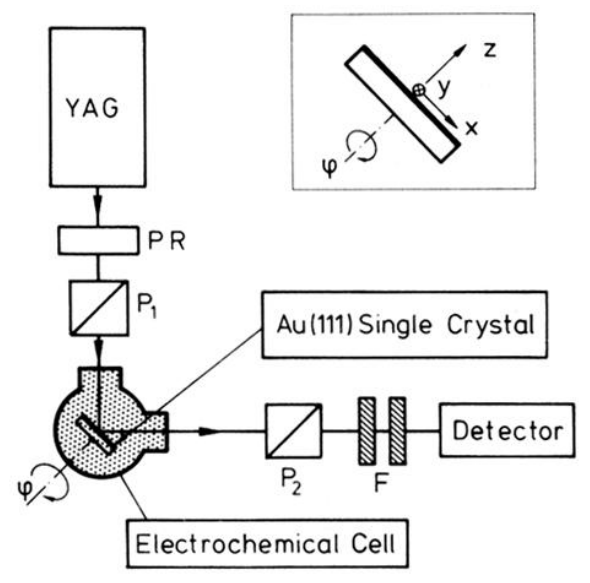

FIG. 1. Experimental setup for SHG observation from Au samples within electrochemical cell. PR: Polarization rotator; $P_{1}, P_{2}$ : polarizers; $F$ : filter for rejection of the fundamental wavelength at $1064 \mathrm{~nm}$. 